\title{
The Principles of Designing an Expert System in Teaching Mathematics
}

\author{
Lailya Salekhova", Albert Nurgaliev, Rinata Zaripova, Nailya Khakimullina \\ Department of Computational Linguistics and Information Systems in Philology, Kazan Federal University, \\ Kazan, 420021, Tatarstan, Russia \\ *Corresponding Author: salekhova2009@rambler.ru
}

Copyright (C) 2013 Horizon Research Publishing All rights reserved.

\begin{abstract}
This study reveals general didactic concepts of the Expert Systems (ES) development process in the educational area. The proof of concept is based on the example of teaching the 8th grade Algebra subject. The main contribution in this work is the implementation of innovative approaches in analysis and processing of data by expert system as well as the development of the step-by-step algorithm to build a table of dependencies between subjects in a course, known as Concept-Effect Relationship (CER) model, which is introduced in this research work. The CER model is the core component of the ES, based on which the system builds the individual educational strategy for a pupil. The problem of building a table of dependencies between subjects in a course has been a significant obstacle when using this approach in real life due to its time complexity and a demand for significant efforts from multiple experts. Our novel approach introduces a general algorithm for building a dependency table with no linking to a specific subject, thus making CER model implementation easier in real-life educational practice.
\end{abstract}

Keywords Expert Systems, Teaching; Mathematics, Education, Multilingualism

\section{Introduction}

With the increasing workload and level of qualification standards of a teacher, arises a problem of fast deployment of innovative technologies in education. The process of new technology deployment can stretch in time due to the fact that its author cannot individually teach a large number of teachers instantly. To cope with this problem we have developed an Expert System (ES) for teaching Mathematics based on multilingualism (Russian-Tatar-English) for the eighth grade pupils. Our innovative approach relies in researching and development of general didactic concepts of defining relationships between levels of correctness of pupils' answers and level understanding of a subject. The ES is capable of consulting a teacher in questions related to the techniques of teaching a subject, based on the analysis of pupils' academic performance. As a result every teacher would be able to receive an expert assistance regardless of his location or time.

The project is represented as a stand-alone application, capable of increasing the effectiveness of a teacher's work. The model of analyzing pupils' academic performance represented in grades is used as a basis for building an individual educational strategy. The system automatically selects the most optimal academic load for each individual pupil as well as for the entire class. The system is capable of analyzing current academic performance of each individual pupil in real time and depending on the improvement or deterioration in pupil performance it differentiates the load of a particular student for instance by generating individual homework exercises. This helps achieving flexibility during developing and realization of the study course.

Due to the fact that some of the responsibilities of the teacher are taken by the ES, the teacher's workload decreases, at the same time increasing the quality of education.

The structure of the paper is as follows: Section 1 presents an overview of the ES, Section 2 discusses the existing technologies in the field of ES, Section 3 presents the principles and architecture for the developed ES, Section 4 demonstrates the features of the developed expert system with the screenshots.

\section{Overview of Expert Systems}

Expert (Latin expertus - experienced) is a person with special knowledge in a particular field of science, art, crafts, etc. The expert with extensive experience has got the skills that allow him to solve complex problems effectively. Consequently, the ES can be defined as a computer program designed to simulate the ability of a human-expert to solve the problem. ES consists of: (a) graphical user interface, (b) knowledge base, (c) databases, (d) inference engine, and (d) working memory (Figure 1) [1]. 


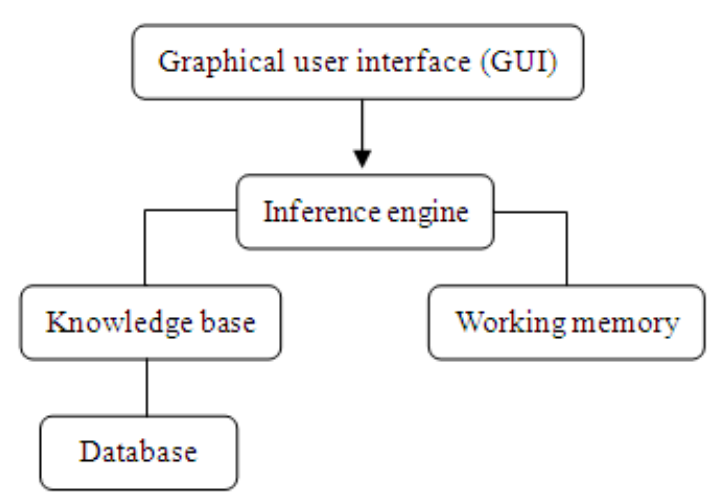

Figure 1. ES components and their interrelationships

The GUI allows a user to interact with the ES. Properly modeled graphical interface is a key factor of increasing the efficiency of using the system. The knowledge base contains all the rules according to which data is processed. The inference engine determines the method, according to which these rules will be used. All logical conclusions drawn from ES are stored in a working memory as long as the appropriate instructions are prompted data from the database.

Inference engine often uses two strategies [1], these are forward and backward chaining. Forward chaining is a top-down method which takes facts as they become available and attempts to draw conclusions (from satisfied conditions in rules) which lead to actions being executed.

Backward chaining is the reverse. It is a bottom-up procedure which starts with goals (or actions) and queries the user about information which may satisfy the conditions contained in the rules. It is a verification process rather than an exploration process.

ES usually is designed to replace a human-expert in cases where: the solution of similar problems can be automated, the expert leaves the job or retires, there is a high cost of hiring an expert, demand for an expert in any time and in remote locations (villages).

\section{The Existing Technologies and Principles}

In recent years, researchers have proposed various approaches for developing adaptive learning systems based on the personal features or learning behaviors of students [2]. Furthermore, models or mechanisms for diagnosing student learning problems and providing personalized learning guidance have been presented as well $[3,4]$.

Among the existing models, the Concept-Effect Relationship (CER) model, which represents the prerequisite relationships among concepts in a course, has been proved to be an effective way of improving the learning performance of students. The CER model demonstrates a systematic procedure for identifying the learning problems of students for each concept taken into account. It has been used to successfully detect the learning problems of students and to give personalized suggestions to them for several science and mathematics courses [5].

Hwang et al. [4] proposed the CER model to represent the prerequisite relationships among concepts that need to be learned in a dedicated order. Such a model has been referred to by several researchers in developing testing and diagnostic mechanisms or systems for improving the learning performance of students. Moreover, various applications have revealed the effectiveness of the CER model.

For example, Jong et al. [5] developed a learning behavior diagnosis system which was applied to a computer course of a university and yielded positive experimental results for both learning status and learning achievement. In the meantime, Tseng et al. [7] employed the CER model to provide learning guidance for individual students in the physics course of a junior high school. Furthermore, Hwang, et al. [4] reported the effectiveness of the CER model in improving the learning achievements of students in a Mathematics course of an elementary school.

In the CER model, the diagnosis of student learning problems mainly depends on the prerequisite relationships between the concepts to be learned. Consider two concepts to be learned, say $C_{i}$ and $C_{j}$. If $C_{i}$ is a prerequisite to efficiently performing the more complex and higher level concept $\mathrm{C}_{\mathrm{j}}$, then a concept-effect relationship $\mathrm{C}_{\mathrm{i}} / \mathrm{C}_{\mathrm{j}}$ is said to exist. For example, to learn the concept "subtraction of positive integer," one may first need to learn "addition of positive integer", while learning "division of positive integer" may require first learning "subtraction of positive integer" and "multiplication of positive integer". For the sake of simplicity let's consider the term "concept" as a topic of a subject.

Figure 2 illustrates an example of a model of the CER for this case. Therefore, if the student does not respond accurately to the majority of questions on the topic «Division of the positive integers», it is likely that the student studied poorly this topic or previous themes «Subtraction of positive integers» and «Multiplication of positive integers» [6]. Thus, teachers can identify problems of students in studying these topics through monitoring of the concept-effect relations.

CER model considers all possible ways for finding the students learning problems. Figure 2 shows two ways to learn the topic $\mathrm{T}_{5}$ on subject,

Path 1: $\mathrm{T}_{1} \rightarrow \mathrm{T}_{2} \rightarrow \mathrm{T}_{3} \rightarrow \mathrm{T}_{5}$

Path 2: $\mathrm{T}_{1} \rightarrow \mathrm{T}_{2} \rightarrow \mathrm{T}_{4} \rightarrow \mathrm{T}_{5}$

In order to provide individual recommendations, coefficients of errors (CE) should be calculated for each student. That requires the creation of a table showing the relation between questions $\left(\mathrm{Q}_{\mathrm{i}}\right)$ in the task and learned topics $\left(T_{j}\right)$. For example, Table 1 shows relation between ten topics and twelve tasks; the value $\left(\mathrm{Q}_{\mathrm{i}}, \mathrm{T}_{\mathrm{j}}\right)$ ranges from 0 to 1 ; «1» indicates an absolute dependency, and « 0 » indicates the lack of any connection between a topic and a task. 


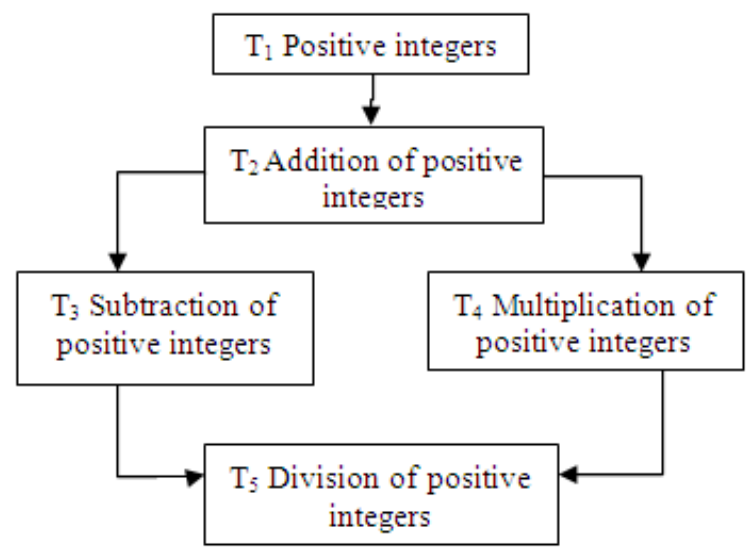

Figure 2. The example of the CER model

CE of a student for topic $T_{j}$ is calculated by dividing the sum of values of $\left(\mathrm{Q}_{\mathrm{i}}, \mathrm{T}_{\mathrm{j}}\right)$ on the tasks where a student made a mistake to the sum of all tasks. Assuming that CE of a student who answered the questions on topics $T_{1}, T_{2}, T_{3}, T_{4}$ and $T_{5}$ are $0.0,0.25,0.35,0.2$ and 0.6 , respectively, we have:

Path 1: $\mathrm{T}_{1}(0.0) \rightarrow \mathrm{T}_{2}(0.25) \rightarrow \mathrm{T}_{3}(0.35) \rightarrow \mathrm{T}_{5}(0.6)$ and

Path 2: $\mathrm{T}_{1}(0.0) \rightarrow \mathrm{T}_{2}(0.25) \rightarrow \mathrm{T}_{4}(0.2) \rightarrow \mathrm{T}_{5}(0.6)$

The threshold value $\theta$ defines the permissible value of CE. If CE $\left(T_{j}\right) \leqslant \theta$, it should be considered that a student has understood the topic $T_{j}$. Otherwise the student has a problem with this topic and it is selected as a root node for building a tree of topics that the student has problems with. Let's consider that $\theta$ equals 0.3 . So our path will be:

Path 1: $\mathrm{T}_{3}(0.35) \rightarrow \mathrm{T}_{5}(0.6)$ and

Path 2: $\mathrm{T}_{5}(0.6)$.

Therefore, the student has a lack of understanding the topics $\mathrm{T}_{3}$ and $\mathrm{T}_{5}$. Moreover, the student has to learn topic $\mathrm{T}_{3}$, before studying $\mathrm{T}_{5}$.

The principles of detecting students' academic performance issues described above will be used as a basis for the development of the ES in studying mathematics.

Table 1. An example of dependencies between exercise questions and topics

\begin{tabular}{|c|c|c|c|c|c|c|c|c|c|c|}
\hline & $\mathrm{T}_{1}$ & $\mathrm{~T}_{2}$ & $\mathrm{~T}_{3}$ & $\mathrm{~T}_{4}$ & $\mathrm{~T}_{5}$ & $\mathrm{~T}_{6}$ & $\mathrm{~T}_{7}$ & $\mathrm{~T}_{8}$ & $\mathrm{~T}_{9}$ & $\mathrm{~T}_{10}$ \\
\hline $\mathrm{Q}_{1}$ & 1 & 0 & 0 & 0.6 & 0 & 0 & 0.2 & 0 & 0 & 0 \\
\hline $\mathrm{Q}_{2}$ & 0 & 0.3 & 0 & 0 & 0 & 1 & 0 & 0 & 0 & 0 \\
\hline $\mathrm{Q}_{3}$ & 0.5 & 0 & 0 & 0 & 0.5 & 0 & 0 & 0 & 0 & 0 \\
\hline $\mathrm{Q}_{4}$ & 0 & 0.4 & 0.2 & 0 & 0 & 0 & 1 & 0.3 & 0.4 & 0 \\
\hline $\mathrm{Q}_{5}$ & 0 & 0 & 0.3 & 1 & 0 & 0 & 0.3 & 0 & 0 & 1 \\
\hline $\mathrm{Q}_{6}$ & 0.3 & 0.4 & 0 & 0.7 & 0.5 & 0 & 0 & 0 & 0 & 0 \\
\hline $\mathrm{Q}_{7}$ & 0 & 0 & 0 & 0 & 0 & 0.4 & 0 & 0 & 0.3 & 0 \\
\hline $\mathrm{Q}_{8}$ & 0.8 & 0 & 0 & 0 & 0 & 0 & 0.6 & 0 & 0 & 0 \\
\hline $\mathrm{Q}_{9}$ & 0.4 & 1 & 0 & 0 & 1 & 0.2 & 0 & 0.8 & 1 & 0.2 \\
\hline $\mathrm{Q}_{10}$ & 0 & 0 & 1 & 0 & 0 & 0 & 0 & 1 & 0 & 0 \\
\hline $\mathrm{Q}_{11}$ & 0 & 0 & 0 & 0 & 0 & 0.5 & 0 & 0 & 0 & 0.6 \\
\hline $\mathrm{Q}_{12}$ & 0 & 0 & 0 & 0.3 & 0 & 0 & 0 & 0 & 0 & 0 \\
\hline
\end{tabular}

\section{The Model of the Expert System for Teaching Mathematics}

Considering the fact that CER model has shown its effectiveness in terms of diagnosing an academic performance of a student, it has been used as a basis for diagnosing the academic performance as well. However in practice determining the dependencies between topics in a course can become time consuming. This problem appears due to the fact that the dependencies between topics are determined by domain experts (e.g. Mathematics teachers with many years of teaching experience). Various teachers might have different opinions regarding the level of dependency between certain topics. Not only it is time consuming for a single expert to define all the dependencies between topics, but also it is quite difficult to integrate various experts' opinions into one dependency table.

We have observed that partially authors of course books take some of the workload of defining the flow of the education process. Therefore using the authors of the course books as the domain experts can simplify the overall process of defining the dependencies between topics. According to this observation we have developed a general methodology for defining the dependencies between topics. This methodology reveals general concepts of defining the dependencies, without aiming to any specific subject. This allows applying it to any course.

Before introducing the methodology few initial conditions and definitions should be made:

1. An atomic topic is a topic which doesn't have any prerequisites for its learning.

2. The definition of the concept-effect relationship should be narrowed down in order to avoid developing of an overly complicated table of dependencies with redundant links. A new definition of a concept-effect relationship would be:

If $\mathrm{C}_{\mathrm{i}}$ is a prerequisite to efficiently performing the more complex and higher level concept $\mathrm{C}_{\mathrm{j}}$, such that doesn't exist a concept $\mathrm{C}_{\mathrm{k}}$ for which $\mathrm{C}_{\mathrm{i}}$ also stands as a prerequisite, at the same time $C_{k}$ is a prerequisite to efficiently performing the concept $C_{j}(1)$. In other words, the dependency can exist between the "neighboring" topics.

$\mathrm{C}_{\mathrm{i}} \rightarrow \mathrm{C}_{\mathrm{j}} \nexists \nexists \mathrm{C}_{\mathrm{k}}: \mathrm{C}_{\mathrm{i}} \rightarrow \mathrm{C}_{\mathrm{k}} \rightarrow \mathrm{C}_{\mathrm{j}}(1)$

3. It was already mentioned that the level of dependency between two topics can vary from 0 to 1 inclusive. If some complex topic has several prerequisites, then let's assume that the sum of all dependencies should be equal to 1 . In addition if there are $\mathrm{N}$ prerequisites for some topic $\mathrm{C}_{\mathrm{j}}$ then each level of dependency should be calculated as $1 / \mathrm{N}$. This is explained by the fact that every topic, no matter how big or small it is, is equally important as other $\mathrm{N}-1$ prerequisite topics, otherwise it contradicts the initial definition of the CER model (Figure 3).

When these initial conditions are met, the step-by-step methodology for developing a dependency table can be described in the following section. For the sake of simplicity and visibility of the process of developing the dependencies between topics, we will use a graph 
representation of a table. Where the root (top) of a graph represent the atomic topic(s) and leafs (bottom) represent the most complex topics is a course that should be learned after learning all other prerequisites.

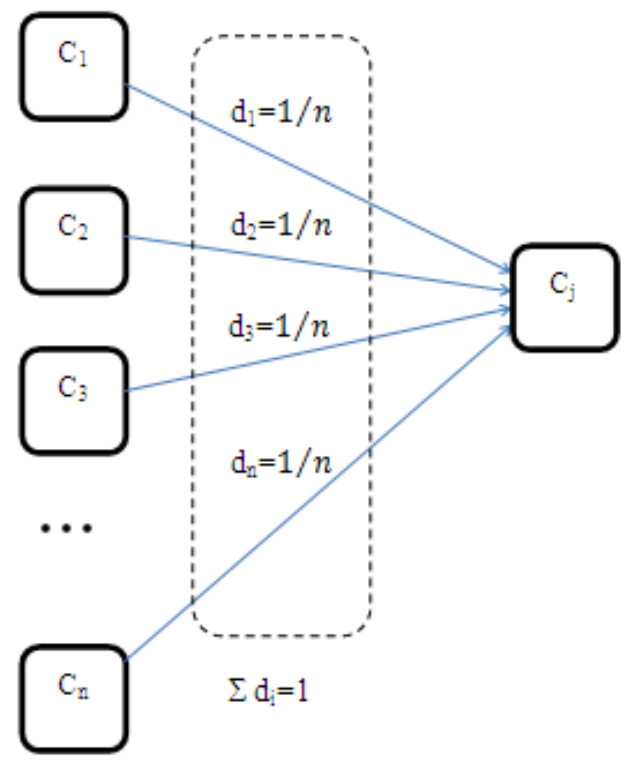

Figure 3. Defining the levels of dependencies between topics.

\subsection{A Methodology for Developing A Dependency Table.}

1. Define the sequence of topics (concepts) $\left[\mathrm{C}_{1}, \mathrm{C}_{2}\right.$, $\mathrm{C}_{3}, \ldots \mathrm{C}_{\mathrm{n}}$ ]. Initial positioning of topics in a course can simplify the process of defining the dependencies of topics, since more complex topics will always be positioned after their prerequisites. A well-defined flow of studied topics allows students to easily learn a subject without jumping from one chapter to another and then back again. In order to accomplish this step three course books, which were approved by the Ministry of Education should be selected. The fact that the book has been approved by the government gives a guarantee that the selected books have a well-defined flow of the topics which allow easy and efficient learning. Referring for three books reduces the risk of following of one author's opinion. In cases when there are various opinions regarding the flow of a course, the most popular one is considered. Therefore a linear dependency of topics obtained from defining their sequence gives a general overview of topics' dependencies, which serves as a basis for further development of the dependency table. This approach provides us the following advantages for optimization of the process of developing the dependency table:

a. Topics, located to the right of the list $\left[\mathrm{C}_{1}, \mathrm{C}_{2}, \mathrm{C}_{3}, \ldots \mathrm{C}_{\mathrm{n}}\right]$ cannot have backward dependencies. In other words, a complex topic cannot be a prerequisite for a simpler topic located to the left of it in the list. Otherwise it contradicts the definition of the CER model. At the same time this fact allows us to search for dependencies between topics only to the right from the examined topic. b. The step 3, which requires determining only the nearest depending topics is simplified, since there is a high probability that the prerequisite topics are located in a few positions away in the list $\left[\mathrm{C}_{1}, \mathrm{C}_{2}, \mathrm{C}_{3}, \ldots \mathrm{C}_{\mathrm{n}}\right]$. Therefore there is no need to traverse the entire list, which significantly reduces time required to fill the entire dependency table.

2. The second step requires determining the atomic topics, according to the following algorithm:

For any $C_{i}$, where $i=1,2,3, \ldots, n$

Compare $C_{i} u C_{j}$, where $j=1,2,3, \ldots, n-1$ and $j<i$

If doesn't exist $C_{j} \rightarrow C_{i}$ for any $j$, then put $C_{i}$ as one of the roots of the graph and remove it from the list $\left[C_{1}, C_{2}, C_{3}, \ldots\right.$ $\left.C_{n}\right]$.

At this stage the atomic topic is determined if there is no prerequisite topic to the left of the considered one. It should be noted that according to the conditions described at the first stage, the first topic in the list $\left[\mathrm{C}_{1}, \mathrm{C}_{2}, \mathrm{C}_{3}, \ldots \mathrm{C}_{\mathrm{n}}\right]$ will always be an atomic topic.

3. The third stage is to determine the nearest depending topics for the considered topic. It is performed according to the following algorithm:

Determine the list of remaining topics: for any $C^{\prime}{ }_{i}$ where $i=1,2, \ldots, k$ and $k<n:\left[C^{\prime}{ }_{1}, C_{2}{ }_{2}, \ldots, C^{\prime}{ }_{k}\right]$;

Determine the list of topics from the lowest level of the dependency graph at the moment: for any $C_{j}$, where $j=1,2, \ldots m$ and $k+m \leq n:\left[C_{1}, C_{2}, \ldots, C_{m}\right]$

Determine the CER: Exists $C j \rightarrow C i$, such that for any $C_{y}$ doesn't exist a CER of the form: $C_{j} \rightarrow C_{y} \rightarrow C_{i}$, where $y$ $=1,2, \ldots, k$ and $y \neq i, y \neq j$.

Put $C_{i}$ under the dependency of $C_{j}$ and remove from the list of remaining topics. There may be several prerequisites for $C_{i}$, in such case the degree of dependency (which varies from 0 to 1), is calculated according to the initial condition 3.

It is required to determine the topics that directly affect successful learning of the considered topic. In order to accomplish this task every concept from the remaining list of topics is checked for dependency among all topics currently located on the leafs of the dependency graph (Figure 4).

Repeat step 3 until the list of the remaining topics gets empty.

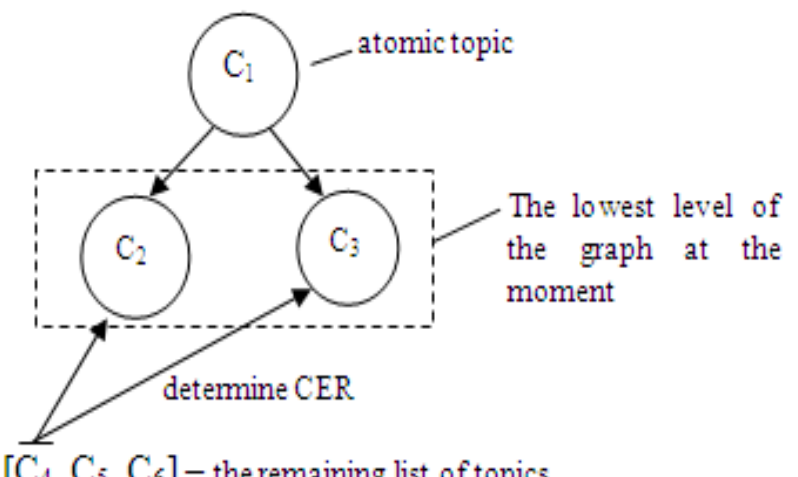

Figure 4. Developing a dependency graph 
The proposed algorithm has been used to develop the dependencies between topics for Algebra course in $8^{\text {th }}$ grades. The architecture of the system is described in Figure 5.

A teacher interacts with a graphical interface which includes such information as academic performance of a student as well as the recommendations for organization of an educational process, generated on the basis of the analysis of incoming data.

The program logic carries out expert analysis and comparison of all the incoming data with the relevant conditions and the expectations that were programmed into the system according to the methodologies of teaching a subject, particularly, mathematics. Based on the conclusions that were made from calculations the system must be able to identify possible gaps in the knowledge of individual student in order to improve their academic performance.

Additional modules connected into the system extend the capabilities of the expert system and adapt it to the specific teacher requirements (for example, a module for automated synchronization with the marks database or a random exercises generator module).

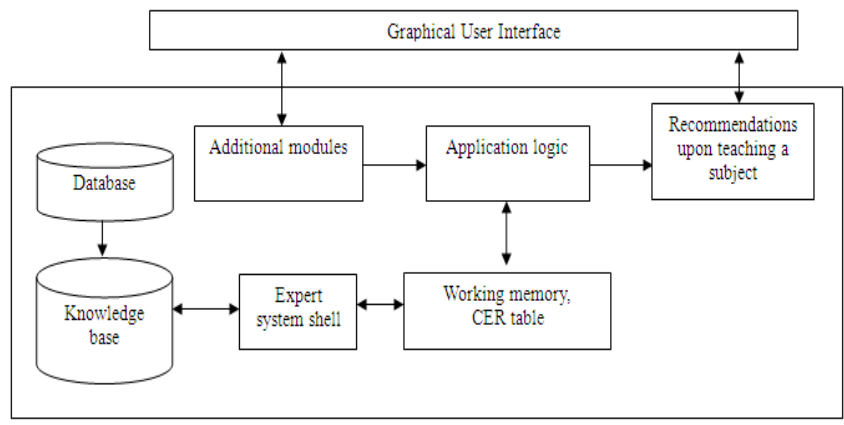

Figure 5. The architecture of the ES for teaching Mathematics

\section{Demonstration}

The main dialog window of the expert system is presented in the Figure 6.

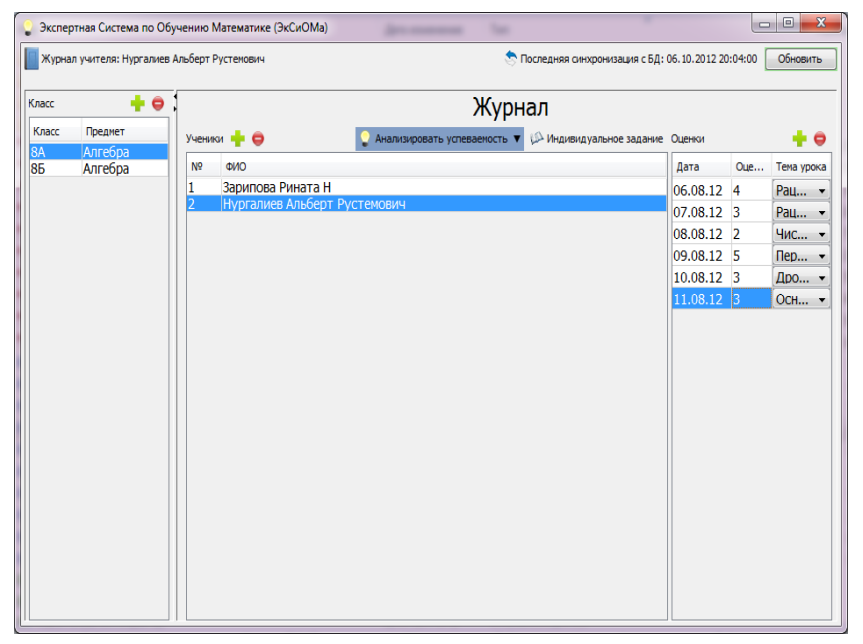

Figure 6. Main window of the ES for teaching Mathematics
Main window of the system was developed with the aim to resemble the appearance of a traditional class journal. The information about the current user is presented on the top of the window. Left column presents the list of the classes where the current teacher teaches his/her subject. When one of the classes is selected from the list, its corresponding list of students is shown in the middle column. By analogy, when one of the students is selected from this list, his/her corresponding grades are displayed along with such information as the date of the grade and topic for which this grade was given. All the lists can be edited using the corresponding buttons on the toolbar above each column.

Main advantage of our system relies in its ability to analyze the academic performance of each student individually as well as of the entire class. The user interface is designed to be intuitive to use by a person with basic knowledge of computer technologies. The analysis of academic performance is accomplished by pressing a single button on a toolbar located on the middle column above the list of students, while the analyzing mode (for the entire class or individual) is done using drop down menu (Figure 7).

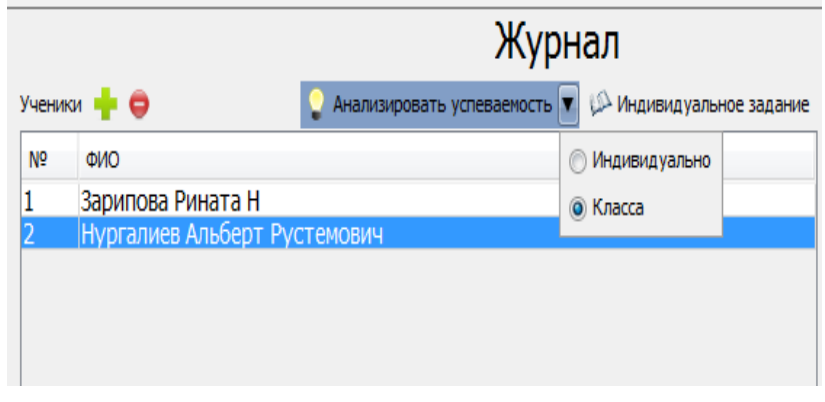

Figure 7. Analyzing feature of the ES for teaching Mathematics

As the result of the analysis for both of these modes would be building of the tree of problematic topics, which represents the weakly learned topics and the order in which they should be learned. For example, if the topic "Fractional expressions" cannot be learned before "Rational expressions", it is not worth trying to eliminate the knowledge gap in a more complex topic "Fractional expressions" when a student has poorly learned a prerequisite topic "Rational expressions". In cases when some problematic topics don't have dependencies between each other, they are displayed as separate roots of a tree (Figure 8). The list of problematic topics for the entire class is defined by analyzing the academic performance of each student in this class with further statistical analysis to determine the most popular problematic topics. If the problematic topic is met in more than the half of the class, it is considered as the knowledge gap for the entire class and is displayed in the resulting tree. 


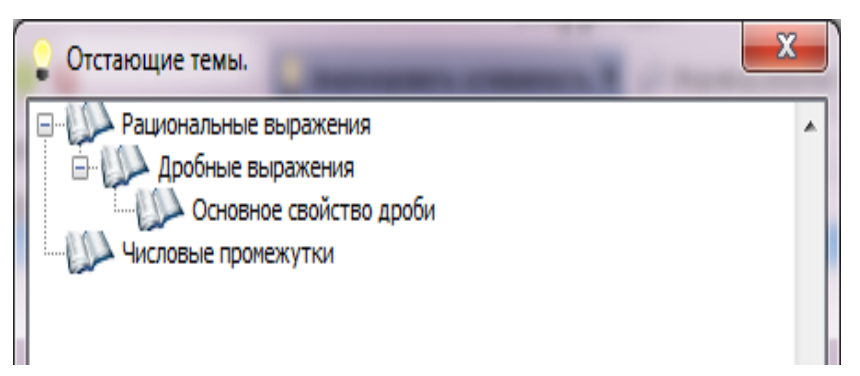

Figure 8. The tree of problematic topics

Based on the analysis of the academic performance of a student, the system is capable of providing individual tasks with the emphasis on the problematic topics. In order to use this feature a user needs to press the button "Individual tasks". The system generates five (by default, but the number can be configured) the most suitable exercises for a student (Figure 9).

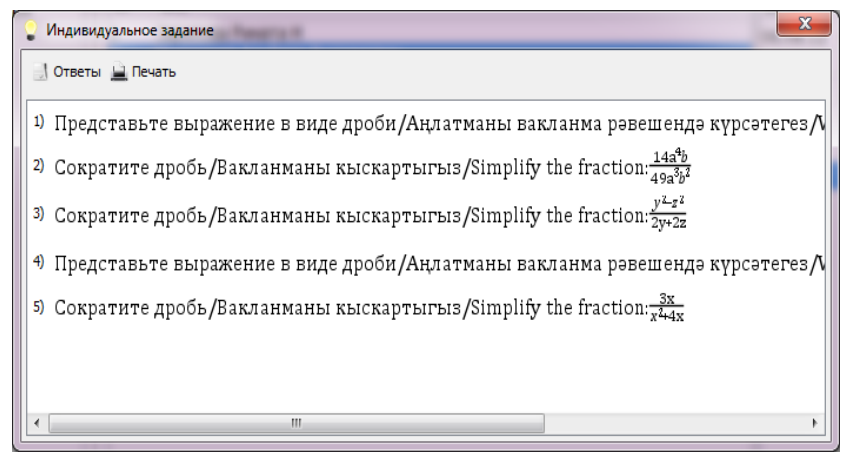

Figure 9. Individual exercises for a student, based on the academic performance analysis.

The exercises can be printed. Also a teacher can see the answers for the tasks (Figure 10).

\begin{tabular}{|l|} 
Ответы \\
1) Печать \\
2) $\frac{p a}{7 \mathrm{~b}} ; \boldsymbol{a} \neq 0, \boldsymbol{b} \neq 0$ \\
3) $\frac{y-z}{2} ; y \neq-z$ \\
4) $\frac{3}{y^{2}}$ \\
5) $\frac{3}{x+4} ; x \neq 0 ; x \neq-4$
\end{tabular}

Figure 10. Answers for the individual exercises

This way a teacher will not face the problem of fast analysis of academic performance of his/her students and will effectively differentiate the academic load in the class. This problem is quite relevant these days, taking into account that in average there are 20-25 students per class and a teacher can teach in up to six classes. Therefore the analysis of academic performance of 120 students can become a time consuming routine work, but nevertheless which doesn't require significant efforts from the teacher.

\section{Conclusion}

Currently the developed ES has been tested for any bugs in the code and the design implementation. They demonstrated that the system is capable of analyzing the academic performance in a simulated environment. As a future work we plan to test the system in real life environment during the next academic year, which starts in September 2013.

According to preliminary results the use of expert systems in education can simplify the process of teaching of subjects, in our case, mathematics. Proper use of the system can significantly reduce the workload of a teacher and leave more time for self-development and improving professional mathematical competence. With the popularization of computer and communication technologies it is very important to create modern system of education in a new information society.

\section{REFERENCES}

[1] M. Patankar, «Rule-based expert system approach to academic advising,» Innovations in Education and Teaching International (IETI), т. 1, № 35, 1998.

[2] C.-Y. Chou, B.-H. Huang and C.-J. Lin, "Complementary machine intelligence and human intelligence in virtual teaching assistant for tutoring program tracing," Computers \& Education, no. 57, 2011.

[3] G. J. Hwang, "A conceptual map model for developing intelligent tutoring systems.," Computers \& Education, vol. 3, no. $40,2003$.

[4] G. J. Hwang, J. C. R. Tseng and G. H. Hwang, "Diagnosing student learning problems based on historical assessment records.," Innovations in Education and Teaching International, vol. 1, no. 45, 2008.

[5] B. S. Jong, T. Y. Chan and Y. L. Wu, "Learning log explorer in e-learning diagnosis.," IEEE Transactions on Education, vol. 3, no. 50, 2007.

[6] P. Panjaburee, G. J. Hwang, W. Triampo and B.-Y. Shih, "A multi-expert approach for developing testing and diagnostic systems based on the concept-effect model," Computers \& Education, no. 55, 2010.

[7] S. S. Tseng, P. C. Sue, J. M. Su, J. F. Weng and W. N. Tsai, "A new approach for constructing the concept map," Computers \& Education, vol. 3, no. 49, 2007. 